\title{
Scale anomaly of a Lifshitz scalar: A universal quantum phase transition to discrete scale invariance
}

\author{
Daniel K. Brattan \\ Interdisciplinary Center for Theoretical Study, University of Science and Technology of China, \\ 96 Jinzhai Road, Anhui, Hefei 230026, People's Republic of China \\ Omrie Ovdat ${ }^{\dagger}$ and Eric Akkermans ${ }^{\ddagger}$ \\ Department of Physics, Technion Israel Institute of Technology, Haifa 3200003, Israel
}

(Received 20 June 2017; published 12 March 2018)

\begin{abstract}
We demonstrate the existence of a universal transition from a continuous scale invariant phase to a discrete scale invariant phase for a class of one-dimensional quantum systems with anisotropic scaling symmetry between space and time. These systems describe a Lifshitz scalar interacting with a background potential. The transition occurs at a critical coupling $\lambda_{c}$ corresponding to a strongly attractive potential.
\end{abstract}

DOI: 10.1103/PhysRevD.97.061701

\section{INTRODUCTION}

Some of the most intriguing phenomena resulting from quantum physics are the violation of classical symmetries, collectively referred to as anomalies [1-4]. One class of anomalies describes the breaking of continuous scale symmetry at the quantum level. A remarkable example of these "scale anomalies" occurs in the case of a nonrelativistic particle in the presence of an attractive, inverse square potential [5-12], which describes the Efimov effect [13-15] and plays a role in various other systems [16-23]. Classically scale invariant [24], any system described by the Hamiltonian, $\hat{H}_{S}=p^{2} / 2 m-\lambda / r^{2}$, exhibits an abrupt transition in the spectrum at $2 m \lambda_{c}=(d-2)^{2} / 4$ [25] where $d$ is the space dimension. For $\lambda<\lambda_{c}$, the spectrum contains no bound states close to $E=0$; however, as $\lambda$ goes above $\lambda_{c}$, an infinite series of bound states appears. Moreover, in this "over-critical" regime, the states arrange themselves in an unanticipated geometric series $E_{n} \propto \exp \left(-2 \pi n / \sqrt{\lambda-\lambda_{c}}\right)$, $n \in \mathbb{Z}$, accumulating at $E=0$. The existence and geometric structure of such levels do not rely on the details of the potential close to its source and is a signature of residual discrete scale invariance since $\left\{E_{n}\right\} \rightarrow\{\exp (-2 \pi /$ $\left.\left.\left.\sqrt{\lambda-\lambda_{c}}\right)\right) E_{n}\right\}=\left\{E_{n}\right\}$. Thus, $H_{S}$ exhibits a quantum phase transition at $\lambda_{c}$ between a continuous scale invariant (CSI) phase and a discrete scale invariant phase (DSI). This

\footnotetext{
*danny.brattan@gmail.com

†somrie@campus.technion.ac.il

¥eric@physics.technion.ac.il
}

Published by the American Physical Society under the terms of the Creative Commons Attribution 4.0 International license. Further distribution of this work must maintain attribution to the author(s) and the published article's title, journal citation, and DOI. Funded by SCOAP. transition has been associated with Berezinskii-KosterlitzThouless (BKT) transitions [12,17,26-30].

Another system - the charged and massless Dirac fermion in an attractive Coulomb potential $\hat{H}_{D}=\gamma^{0} \gamma^{j} p_{j}-$ $\lambda / r$ [31] —also belongs to the same universal class of systems with these abrupt transitions. The similarity between the spectra and transition of these Dirac and Schrödinger Hamiltonians motivates the study of whether a transition of this sort is possible for a generic scale invariant system. Specifically, these different Hamiltonians share a similar property - the power law form of the corresponding potential matches the order of the kinetic term. In this paper, we examine whether this property is a sufficient ingredient by considering a generalized class of one-dimensional Hamiltonians,

$$
\hat{H}_{N}=\left(p^{2}\right)^{N}-\frac{\lambda_{N}}{x^{2 N}}
$$

where $N$ is an integer and $\lambda_{N}$ a real coupling, and study if they exhibit a transition of the same universality class as $\hat{H}_{S}=\hat{H}_{1}, \hat{H}_{D}$.

Hamiltonian (1) describes a system with non-quadratic anisotropic scaling between space and time for $N>1$. This "Lifshitz scaling symmetry" [32], manifest in (1), can be seen for example at the finite temperature multicritical points of certain materials [33,34] or in strongly correlated electron systems [35-37]. It may also have applications in particle physics [32], cosmology [38] and quantum gravity [39-43]. The non-interacting mode $\left(\lambda_{N}=0\right)$ can also appear very generically, for example in non-relativistic systems with spontaneous symmetry breaking [44].

Generalizing (1) to higher-dimensional flat or curved spacetimes also introduces intermediate scale invariant terms which are products of radial derivatives and powers 
of the inverse radius. For $d>1$ the potential in (1) can be generated by considering a Lifshitz scale invariant system with charge and turning on a background gauge field [32,45-47] consisting of the appropriate multipole moment. The procedures we use throughout this paper are readily extended to these situations and the simple model (1) is sufficient to capture the desired features.

Our main results are summarized as follows. In accordance with the $N=1$ case, there is a quantum phase transition at $\lambda_{N, c} \equiv(2 N-1) ! !^{2} / 2^{2 N}$ for all $N>1$ from a CSI phase to a DSI phase in the low energy regime $|E|^{1 / 2 N} x_{0} \ll 1$, where $x_{0}>0$ is a short distance cutoff. The CSI phase contains no bound states and the DSI phase is characterized by an infinite set of bound states forming a geometric series as given by Eq. (11). The transition and $\lambda_{N, c}$ value are independent of the short distance physics characterized by the boundary condition at $x=x_{0}$. For $\left(\lambda_{N}-\lambda_{N, c}\right) \rightarrow 0^{+}$, the analytic behavior of the spectrum is characteristic of the BKT scaling in analogy with $N=1$ $[12,17]$ and as shown by Eq. (17). We analyze the $x_{0}=0$ case, obtain its self adjoint extensions and spectrum and obtain similar results.

\section{THE MODEL}

Corresponding to (1) is the action of a complex scalar field in $(1+1)$ dimensions:

$$
\int d t \int_{x=x_{0}}^{\infty} d x \frac{i}{2}\left(\Psi^{*} \partial_{t} \Psi-\text { c.c. }\right)-\left|\partial_{x}^{N} \Psi\right|^{2}+\frac{\lambda_{N}}{x^{2 N}}|\Psi|^{2},
$$

where c.c. indicates the complex conjugate. This field theory has manifest Lifshitz scaling symmetry, $(t, x) \mapsto$ $\left(\Lambda^{2 N} t, \Lambda x\right)$ when $x_{0} \rightarrow 0$. The scaling exponent of $\Lambda^{2}$ is called the "dynamical exponent" and has value $N$ in this case. The action represents a Lifshitz scalar with a single time derivative and can be recovered as the low energy with respect to mass limit of a charged, massive Lifshitz scalar which is quadratic in time derivatives [48]. The eigenstates of Hamiltonian (1) are given by stationary solutions of the subsequent equations of motion [49].

Consider the case $x_{0}<x<\infty$ with $x_{0}=0$. The classical scaling symmetry of (1) implies that if there is one negative energy bound state then there is an unbounded continuum. Thus, the Hamiltonian is non-self-adjoint [51,52]. The origin of this phenomenon, already known from the $N=1$ case, is the strong singularity of the potential at $x=0$. To remedy this problem, the operator can be made self-adjoint by applying boundary conditions on the elements of the Hilbert space through the procedure of selfadjoint extension [53]. Alternatively, a suitable cutoff regularization at $x_{0}>0$ can be chosen to ensure selfadjointness as well as bound the spectrum from below by an intrinsic scale [8] leaving some approximate DSI at low energies.
For $N=1$ in (1), the continuous scaling invariance of $\hat{H}_{1}$ is broken anomalously as a result of restoring selfadjointness. In particular, for $\lambda_{1}>1 / 4$, one obtains an energy spectrum given by $E_{1}=-E_{0} \exp \left(-(2 \pi n) / \sqrt{\lambda_{1}-\lambda_{c}}\right)$ for $n \in \mathbb{Z}$. The energies are related by a discrete family of scalings which manifest a leftover DSI in the system.

In what follows, we shall demonstrate that in the case of large positive $\lambda_{N}$ (attractive potential) one finds, for all $N$, a regime with a geometric tower of states using the method of self-adjoint extension. Subsequently, we bound the resulting spectrum from below, while maintaining DSI at low energies, by keeping $x_{0}>0$ and applying generic (selfadjoint) boundary conditions at a cutoff point. We obtain that a transition from CSI to DSI is a generic feature in the landscape of these Hamiltonians (1), independent of the choice of boundary condition and in a complete analogy with the $N=1$ case [5-12]. By analytically solving the eigenvalue problem for all $N$, we obtain an expression for the critical $\lambda_{N}$ and for the resulting DSI spectrum in the over-critical regime.

\section{SELF-ADJOINT EXTENSION}

To determine whether the Hamiltonians (1) can be made self-adjoint one can apply von Neumann's procedure [51-55]. This consists of counting the normalizable solutions to the energy eigenvalue equation with unit imaginary energies of both signs. When there are $M$ linearly independent solutions of positive and negative sign, respectively, there is a $U(M)$ parameter family of conditions at $x=0$ that can make the operator self-adjoint. If $M=0$, it is essentially self-adjoint and if the number of positive and negative solutions do not match then the operator cannot be made self-adjoint.

For every $N$, there are $N$ exponentially decaying solutions at infinity for $E= \pm i$. Normalizability then depends on their $x \rightarrow 0$ behavior which requires a complete solution of the eigenfunctions. The energy eigenvalue equation from (1) has an analytic solution in terms of generalized hypergeometric functions [56] (see the Supplemental material [57]) for arbitrary complex energy and $\lambda_{N}$. Near $x=0$, the analytic solutions are characterized by the roots of the indicial equation,

$$
\lambda_{N}=(-1)^{N} \Delta(\Delta-1)(\Delta-2) \ldots(\Delta-2 N+1),
$$

which can be obtained by inserting $\Psi(x)=x^{\Delta}\left(1+\mathcal{O}\left(x^{2 N}\right)\right)$ into the eigenvalue equation and solving for $\Delta$. We label the $2 N$ solutions of (2) as $\Delta_{i}, i=1, \ldots, 2 N$ and order by ascending real part followed by ascending imaginary part. Since (2) is real and symmetric about $N-1 / 2$, all roots can be collected into pairs which sum to $2 N-1$ (see Fig. 1). In addition, because $N-1 / 2 \geq-1 / 2$, there can be at most $N$ roots with $\operatorname{Re}\left(\Delta_{i}\right) \leq-1 / 2$. Thus, near $x=0$, a generic combination of the $N$ decaying solutions has the form 


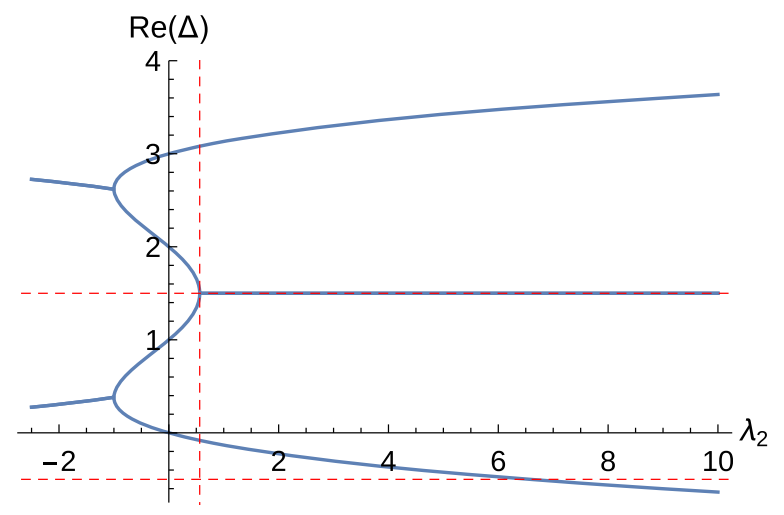

FIG. 1. A plot of the real part of the solution to the indicial equation (2) for $N=2$ against $\lambda_{2}$. The dotted, red vertical line indicates where $\lambda_{2}=9 / 16$ above which the first pair of complex roots appears. The lowermost horizontal red line indicates where one of the roots becomes non-normalizable at positive $\lambda_{2}$ (i.e. $\lambda_{2}=105 / 16$ ) while the uppermost indicates the symmetry line responsible for pairing roots i.e. $\Delta=3 / 2$.

$$
\Psi(x)=\sum_{i=1}^{N}\left[(\epsilon x)^{\Delta_{i}} \phi_{i}+\cdots+(\epsilon x)^{\Delta_{i+N}} O_{i}+\ldots\right],
$$

where $E$ is the energy, $\epsilon \equiv|E|^{1 / 2 N}$ and $\phi_{i}, O_{i}$ are complex numbers [58]. We have distinguished modes with the potential to be divergent as $x \rightarrow 0$ by the coefficients $\phi_{i}$. Taking ratios of $O_{i}$ and $\phi_{i}$ yields $N$-independent dimensionless scales.

To obtain the number of independent normalizable solutions $M$, we need to count the number $m$ of roots $\Delta_{i}$ with real parts less than $-1 / 2$ as $\lambda_{N}$ varies. When any root violates this bound we must take a linear combination of our $N$ decaying functions to remove it from the near origin expansion and as a result $M=N-m$. This is easily accomplished by examining the analytic solutions in an expansion about $x=0$.

Generically, for $\lambda_{N}$ small in magnitude, $m=0$, and there is an $U(N)$ self-adjoint extension [59]. As $\lambda_{N}$ increases in absolute value and the real part of some roots go below $-1 / 2$ the dimension of the self-adjoint parameter decreases. Eventually $\hat{H}_{N}$ either becomes essentially selfadjoint (negative $\lambda_{N}$, repulsive potential) or has a $U(1)$ self-adjoint extension (positive $\lambda_{N}$, attractive potential). The different regimes are summarized for $N=1,2,3$ in Table I.
Now that we have determined when our operator can be made self-adjoint we would like to obtain its energy spectrum. To that purpose, we define the boundary form, $[\Psi, \Phi](x)$, by computing the difference $\hat{H}_{N}-\hat{H}_{N}^{\dagger}$ :

$$
\begin{gathered}
\left\langle\Phi\left|\hat{H}_{N}\right| \Psi\right\rangle-\left\langle\Phi\left|\hat{H}_{N}^{\dagger}\right| \Psi\right\rangle=[\Phi, \Psi](\infty)-[\Phi, \Psi]\left(x_{0}\right), \\
\left\langle\Phi\left|\hat{H}_{N}\right| \Psi\right\rangle=\int_{x=x_{0}}^{\infty} d x \Phi^{*}(x) \hat{H}_{N} \Psi(x),
\end{gathered}
$$

where

$$
[\Phi, \Psi](x)=\sum_{j=1}^{2 N}(-1)^{N+j-1} d_{x}^{j-1} \Phi^{*}(x) d_{x}^{2 N-j} \Psi(x)
$$

and the boundary form is calculated by moving the derivatives in $\left\langle\Phi\left|\hat{H}_{N}\right| \Psi\right\rangle$ to obtain $\left\langle\Phi\left|\hat{H}_{N}^{\dagger}\right| \Psi\right\rangle$ (see Supplemental material [57]). Setting $\Phi=\Psi$ and using the time-dependent Schrödinger equation, it is straightforward to show that

$$
\partial_{t} \rho=\partial_{x}[\Psi, \Psi], \quad \rho=|\Psi|^{2},
$$

so that the boundary form can be interpreted as the value of the probability current at $x=x_{0}$.

We are interested in evaluating (4) on the energy eigenfunctions and require it to vanish when taking $x_{0} \rightarrow 0$ as this fixes any remaining free parameters of a general solution. For $E<0$ there are $N$ exponentially decaying solutions at infinity. A general energy eigenfunction is a sum of these and thus the boundary form (4) evaluated at infinity is zero. For $\hat{H}_{N}$ to be self-adjoint it is necessary to impose the same boundary conditions on the wave functions and their adjoints while ensuring that (6) vanishes.

By examining (2), it can be seen that for $\lambda_{N}>\lambda_{N, c} \equiv$ $(2 N-1) ! !^{2} / 2^{2 N}$ there is a pair of complex roots whose real part is fixed to be $N-1 / 2$, i.e.

$$
\begin{gathered}
\Delta_{N}=\Delta_{N+1}^{*}=(N-1 / 2)-i \nu_{N}\left(\lambda_{N}\right), \\
\nu_{N}\left(\lambda_{N}\right)=\sqrt{\lambda_{N}-\lambda_{N, c}}\left(\alpha_{N}^{-1}+\mathcal{O}\left(\lambda_{N}-\lambda_{N, c}\right)\right),
\end{gathered}
$$

\begin{tabular}{|c|c|c|c|c|c|c|}
\hline \multirow{2}{*}{$\begin{array}{l}N \\
\lambda \\
\end{array}$} & \multicolumn{2}{|c|}{1} & \multicolumn{2}{|c|}{2} & \multicolumn{2}{|r|}{3} \\
\hline & $-\frac{3}{4}<\lambda_{1}$ & $\lambda_{1}<-\frac{3}{4}$ & $\frac{105}{16}<\lambda_{2}$ & $-45<\lambda_{2}<\frac{105}{16}$ & $693 \lesssim \lambda_{3}$ & $-162 \lesssim \lambda_{3} \lesssim 693$ \\
\hline Extension & $U(1)$ & Self-adjoint & $U(1)$ & $U(2)$ & $U(1)$ & $U(3)$ \\
\hline
\end{tabular}

where $\alpha_{N}$ is a constant given explicitly in the Supplemental material [57]. At sufficiently positive $\lambda_{N}, \Delta_{1}, \ldots, \Delta_{N-1}$ are

TABLE I. The regimes of self-adjoint extension parameter with $N=1,2,3$ for some values of $\lambda_{N}$. The bounds for $N=1,2$ are exact while those for $N=3$ are approximate and determined by numerically solving the indicial Eq. (2). A table showing $\lambda_{N}$, for $N=1,2,3$, to larger negative values can be found in the Supplemental material [57]. 
not normalizable and the pair (8) are the leading normalizable roots. This is illustrated in Fig. 1 for $N=2$. Removing the $N-1$ non-normalizable roots yields a single decaying wave function with arbitrary energy. As such, (3) becomes

$$
\begin{aligned}
\Psi(x)= & \phi_{N}(\epsilon x)^{N-1 / 2-i \nu_{N}}+O_{1}(\epsilon x)^{N-1 / 2+i \nu_{N}} \\
& +\sum_{j \geq 2} O_{j}(\epsilon x)^{\Delta_{j+N}}+\ldots,
\end{aligned}
$$

where the $N$ dimensionless and energy-independent scales, $O_{j} / \phi_{N}$, are now fixed. Substituting (10) for two energies $E$ and $\tilde{E}$ into (6) yields a term $\propto\left(|E / \tilde{E}|^{2 i \nu_{N} / N}-\left|O_{1} / \phi_{N}\right|^{2}\right)$ as $x_{0} \rightarrow 0$. Thus, the self-adjoint boundary condition is equivalently an energy quantization condition relating an arbitrary reference energy $E_{0}>0$ to a geometric tower of energies:

$$
E_{n}=-E_{0} e^{-\frac{N \pi n}{\nu_{N}}}, \quad n \in Z
$$

The reference energy $E_{0}$ is a free parameter and can be chosen arbitrarily.

\section{CUTOFF REGULARIZATION}

We shall now consider $x_{0}>0$ and choose boundary conditions to give a lower bound to the energy spectrum while preserving approximate DSI near zero energy. We will impose the most general boundary condition on the cutoff point consistent with unitary time evolution [60]. As the $M=N$ decaying solutions are finite at all points $x_{0}>0$, independent of $\lambda_{N}$, this general boundary condition corresponds to an $U(N)$ self-adjoint extension.

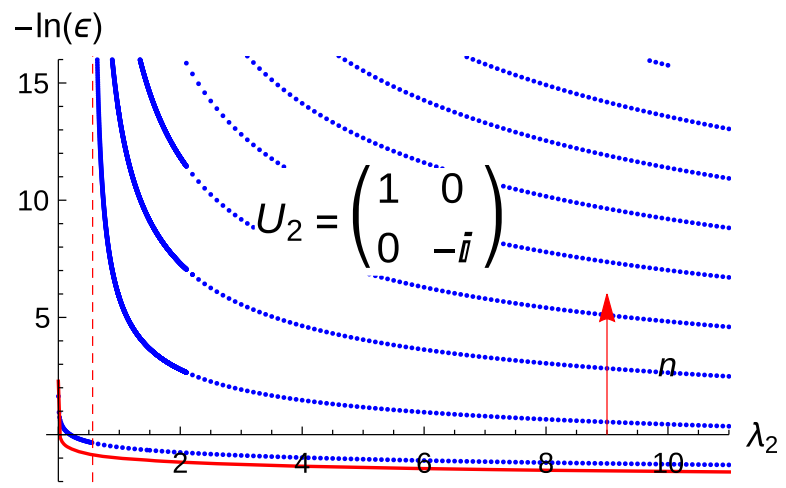

FIG. 2. A plot of the logarithm of $\epsilon$ against $\lambda_{2}$ for a cutoff position $x_{0}=e^{-1}$ and boundary condition displayed. For $\lambda_{2}<$ $\lambda_{2, c}=9 / 16$ there are no bound states satisfying $\epsilon x_{0} \ll 1$ (although there is an isolated bound state outside this energy bound). The solid red line indicates the lower bound on the negative energies as discussed in the Supplemental material [57]. The dotted red line at $\lambda_{2, c}$ indicates where the first pair of complex roots appears, above which we can see the geometric tower abruptly appearing from $\epsilon=0$.
To obtain the boundary condition, we diagonalize (6) by defining $\Psi_{k}^{ \pm}\left(x_{0}\right)$,

$$
\begin{gathered}
x_{0}^{k-1} d_{x}^{k-1} \Psi\left(x_{0}\right)=\Psi_{k}^{+}\left(x_{0}\right)+\Psi_{k}^{-}\left(x_{0}\right), \\
x_{0}^{2 N-k} d_{x}^{2 N-k} \Psi\left(x_{0}\right)=e^{i \pi\left(k-\frac{1}{2}\right)}\left[\Psi_{k}^{+}\left(x_{0}\right)-\Psi_{k}^{-}\left(x_{0}\right)\right]
\end{gathered}
$$

for $1 \leq k \leq N$. After this redefinition, (6) evaluated at $x=x_{0}$ becomes proportional to

$$
\boldsymbol{\Phi}^{+}\left(x_{0}\right)^{\dagger} \cdot \boldsymbol{\Psi}^{+}\left(x_{0}\right)-\boldsymbol{\Phi}^{-}\left(x_{0}\right)^{\dagger} \cdot \boldsymbol{\Psi}^{-}\left(x_{0}\right) .
$$

The vanishing of (14) can be achieved by setting

$$
\boldsymbol{\Psi}^{+}\left(x_{0}\right)=U_{N} \boldsymbol{\Psi}^{-}\left(x_{0}\right),
$$

for some arbitrary unitary matrix: $U_{N}[53,61]$. This additionally ensures that elements of the space of wave functions and its adjoint have the same boundary conditions, making $\hat{H}_{N}$ self-adjoint on said space. The matrix $U_{N}$ can only be specified by supplying additional physical information beyond the form of the Hamiltonian.

As an illustration of the appearance of the geometric tower at $N>1$, consider Figs. 2 and 3. The former plots $\epsilon$ for $N=2$ against $\lambda_{2}$. It is plain that as soon as $\lambda_{2}>9 / 16$ (the dotted red line) there is a sudden transition from no bound states satisfying $\epsilon \ll x_{0}^{-1}$ (and one isolated bound state), to a tower of states. Similarly, Fig. 3 plots the logarithm of $E_{n} / E_{n+1}$ for $N=3$ as a function of $\lambda_{3}$ at low $\epsilon x_{0}$. The result, shown by the blue points in Fig. 3, is a good match with $\pi / \nu_{3}$ with $\nu_{3}$ defined by (8).

For general $N, \lambda_{N}>\lambda_{N, c}$ and small enough energies, we shall now argue that one always finds DSI with the scaling defined in (11) using a small $\epsilon$ expansion. Determining the

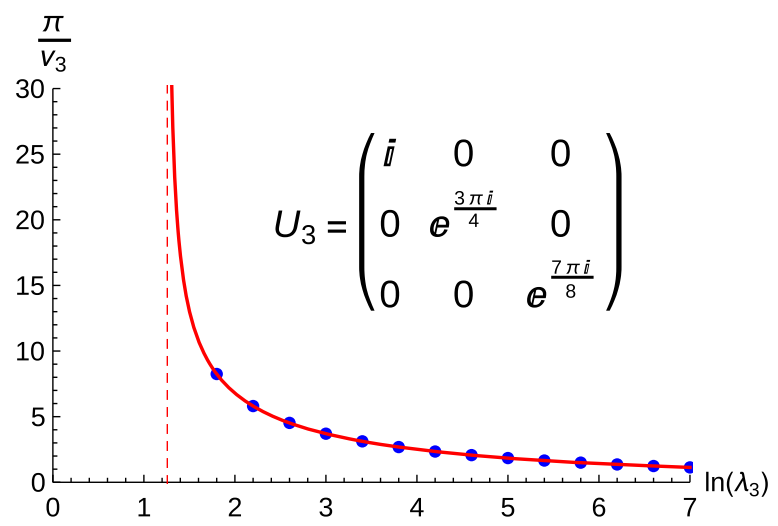

FIG. 3. A plot of $\pi / \nu_{3}$ against $\ln \lambda_{3}$ for a cutoff position $x_{0}=e^{-1}$ and boundary condition displayed. The solid red line indicates the numerical result from solving (2) for the roots defined in (8). The blue dots are calculated by numerically determining the gradient of $\ln E_{n} / E_{n+1}$ against $n$ for several $n$ corresponding to $\epsilon x_{0} \ll 1$. The red dotted line indicates the critical $\lambda_{3}$. 
energy eigenstates analytically for arbitrary boundary conditions is made difficult for $N>1$ due to the presence of multiple distinct complex roots in the small energy expansion. However, we shall show that one pair makes a contribution that decays more slowly as $\epsilon \rightarrow 0$ than any other and derive an approximation in this limit.

Consider (3) evaluated at $x \sim x_{0}$ which is a good approximation to the wave function when $\epsilon x_{0} \ll 1$. Imposing decay fixes $N$ of the $2 N$ free parameters. Without loss of generality we can take them to be $O_{i}$ so that $O_{i}=G_{i}{ }^{j} \phi_{j}$ for some complex $(N \times N)$-matrix $G$. The $N$ remaining free parameters $\phi_{i}$ will be fixed by wave function normalization and boundary conditions at the cutoff point (15). The generic dependence of $\phi_{i}$ on $\epsilon, x_{0}$ for $\epsilon x_{0} \ll 1$ is extractable. Applying the redefinition $\tilde{\phi}_{i} \equiv \phi_{i}\left(\epsilon x_{0}\right)^{\Delta_{i}}$ implies

$$
O_{i}\left(\epsilon x_{0}\right)^{\Delta_{i+N}} \rightarrow G_{i}^{j}\left(\epsilon x_{0}\right)^{\Delta_{i+N}-\Delta_{j}} \tilde{\phi}_{j} .
$$

Given our canonical ordering of the roots and that $\lambda>\lambda_{c}$ we have $\operatorname{Re}\left(\Delta_{i+N}-\Delta_{j}\right) \geq 0$ with equality only when $i=1$ and $j=N$. For $\epsilon x_{0} \ll 1$ the leading contributions to the wave function (3) have the form

$$
\Psi(x)=\sum_{i=1}^{N} \tilde{\phi}_{i}\left(\frac{x}{x_{0}}\right)^{\Delta_{i}}+G_{1}{ }^{N} \tilde{\phi}_{N}\left(\epsilon x_{0}\right)^{2 i \nu_{N}}\left(\frac{x}{x_{0}}\right)^{\Delta_{N+1}}
$$

where $\epsilon x_{0}$ only enters the leading term through a phase and all other contributions to $O_{i}$ from the $\tilde{\phi}_{i}$ drop out as they come with $\epsilon x_{0}$ to a real positive power. The displayed terms above are the relevant ones at low energies for solving (15).
Moreover these leading terms are invariant under the discrete scaling transformation and thus we have DSI. As a result, applying (15) will necessarily give the energy spectrum (11) for $\epsilon x_{0} \ll 1$ with $E_{0}$ a number depending on $U(N)$ and the cutoff $x_{0}$.

We can use our expression (9) for $\nu_{N}$ in terms of $\lambda_{N}$ to find:

$$
\frac{E_{n}}{E_{0}}=-\exp \left(-\frac{N \pi n \alpha_{N}}{\sqrt{\lambda_{N}-\lambda_{N, c}}\left(1+\mathcal{O}\left(\lambda_{N}-\lambda_{N, c}\right)\right)}\right),
$$

characteristic of the BKT scaling for $\lambda_{N} \rightarrow \lambda_{N, c}$.

With the above considerations we can say that a CSI to DSI transition, at $\epsilon x_{0} \ll 1$, is a generic feature of our models is for the consequences of DSI to be relevantindependent of the completion of the potential near the origin. This is in complete analogy with the $N=1$ case. Thus, the Hamiltonian (1) need only be effective for the consequences of DSI to be relevant.

\section{ACKNOWLEDGMENTS}

The work of D. K. B. was supported in part by the Israel Science Foundation under Grant No. 504/13 and is currently supported by a key grant from the National Science Foundation of China with Grant No. 11235010. This work was also supported by the Israel Science Foundation Grant No. 924/09. D. K. B. would like to thank the Technion and University of Haifa at Oranim for their support. D. K. B. would also like to thank Matteo Baggioli and Yicen Mou for reading early drafts.
[1] S. L. Adler, Phys. Rev. 177, 2426 (1969).

[2] J. S. Bell and R. Jackiw, Nuovo Cimento A 60, 47 (1969).

[3] J. G. Esteve, Phys. Rev. D 34, 674 (1986).

[4] B. R. Holstein, Am. J. Phys. 61, 142 (1993).

[5] K. M. Case, Phys. Rev. 80, 797 (1950).

[6] V. de Alfaro, S. Fubini, and G. Furlan, Nuovo Cimento Soc. Ital. Fis. 34, 569 (1976).

[7] L. D. Landau, Quantum Mechanics: Non-relativistic Theory (Butterworth-Heinemann, Oxford, 1991).

[8] H. E. Camblong, L. N. Epele, H. Fanchiotti, and C. A. G. Canal, Phys. Rev. Lett. 85, 1590 (2000).

[9] G. N. J. Añaños, H. E. Camblong, and C. R. Ordóñez, Phys. Rev. D 68, 025006 (2003).

[10] H. W. Hammer and B. G. Swingle, Ann. Phys. (N.Y.) 321, 306 (2006).

[11] E. Braaten and D. Phillips, Phys. Rev. A 70, 052111 (2004).

[12] D. B. Kaplan, J.-W. Lee, D. T. Son, and M. A. Stephanov, Phys. Rev. D 80, 125005 (2009).

[13] V. Efimov, Phys. Lett. B 33, 563 (1970).
[14] V. Efimov, Sov. J. Nucl. Phys. 12, 589 (1971).

[15] E. Braaten and H.-W. Hammer, Phys. Rep. 428, 259 (2006).

[16] J.-M. Lévy-Leblond, Phys. Rev. 153, 1 (1967).

[17] E. B. Kolomeisky and J. P. Straley, Phys. Rev. B 46, 12664 (1992).

[18] K. S. Gupta and S. G. Rajeev, Phys. Rev. D 48, 5940 (1993).

[19] H. E. Camblong, L. N. Epele, H. Fanchiotti, and C. A. G. Canal, Phys. Rev. Lett. 87, 220402 (2001).

[20] C. Nisoli and A. R. Bishop, Phys. Rev. Lett. 112, 070401 (2014).

[21] T. R. Govindarajan, V. Suneeta, and S. Vaidya, Nucl. Phys. B583, 291 (2000).

[22] H. E. Camblong and C. R. Ordonez, Phys. Rev. D 68, 125013 (2003).

[23] S. Bellucci, A. Galajinsky, E. Ivanov, and S. Krivonos, Phys. Lett. B 555, 99 (2003).

[24] R. W. Jackiw, Diverse Topics in Theoretical and Mathematical Physics (World Scientific, Singapore, 1995). 
[25] Thus, for $d=2$, an attractive potential is always overcritical.

[26] K. Jensen, A. Karch, D. T. Son, and E. G. Thompson, Phys. Rev. Lett. 105, 041601 (2010).

[27] K. Jensen, Phys. Rev. D 82, 046005 (2010).

[28] K. Jensen, Phys. Rev. Lett. 107, 231601 (2011).

[29] B. Derrida and M. Retaux, J. Stat. Phys. 156, 268 (2014).

[30] H. Gies and G. Torgrimsson, Phys. Rev. Lett. 116, 090406 (2016).

[31] O. Ovdat, J. Mao, Y. Jiang, E. Y. Andrei, and E. Akkermans, Nat. Commun. 8, 507 (2017).

[32] J. Alexandre, Int. J. Mod. Phys. A 26, 4523 (2011).

[33] R. M. Hornreich, M. Luban, and S. Shtrikman, Phys. Rev. Lett. 35, 1678 (1975).

[34] G. Grinstein, Phys. Rev. B 23, 4615 (1981).

[35] E. Fradkin, D. A. Huse, R. Moessner, V. Oganesyan, and S. L. Sondhi, Phys. Rev. B 69, 224415 (2004).

[36] A. Vishwanath, L. Balents, and T. Senthil, Phys. Rev. B 69, 224416 (2004).

[37] E. Ardonne, P. Fendley, and E. Fradkin, Ann. Phys. (Amsterdam) 310, 493 (2004).

[38] S. Mukohyama, Classical Quantum Gravity 27, 223101 (2010).

[39] M. Reuter, Phys. Rev. D 57, 971 (1998).

[40] S. Kachru, X. Liu, and M. Mulligan, Phys. Rev. D 78, 106005 (2008).

[41] P. Horava, Phys. Rev. Lett. 102, 161301 (2009).

[42] P. Horava, Phys. Rev. D 79, 084008 (2009).

[43] H. Gies, B. Knorr, S. Lippoldt, and F. Saueressig, Phys. Rev. Lett. 116, 211302 (2016).

[44] T. Brauner, Symmetry 2, 609 (2010).

[45] P. Horava, Phys. Lett. B 694, 172 (2010).

[46] S. R. Das and G. Murthy, Phys. Rev. Lett. 104, 181601 (2010).

[47] C. F. Farias, M. Gomes, J. R. Nascimento, A. Yu. Petrov, and A. J. da Silva, Phys. Rev. D 85, 127701 (2012).

[48] S. Pal and B. Grinstein, J. High Energy Phys. 12 (2016) 012.

[49] Additional boundary terms will be required to make the variation of the action vanish on boundary conditions that make the corresponding Hamiltonian operator self-adjoint [50].

[50] M. Asorey, D. Garcia-Alvarez, and J. M. Munoz-Castaneda, in Proceedings of 7th Workshop on Quantum Field Theory Under the Influence of External Conditions (QFEXT 05) Barcelona, Catalonia, Spain, J. Phys. A 39, 6127 (2006).

[51] G. Bonneau, J. Faraut, and G. Valent, Am. J. Phys. 69, 322 (2001).

[52] A. Ibort, F. Lledó, and J. M. Pérez-Pardo, Ann. Inst. Henri Poincaré 16, 2367 (2015).

[53] D. M. Gitman, I. V. Tyutin, and B. L. Voronov, arXiv: 0903.5277.

[54] K. Meetz, Nuovo Cimento 34, 690 (1964).

[55] D. M. Gitman, I. Tyutin, and B. L. Voronov, Self-adjoint Extensions in Quantum Mechanics: General Theory and Applications to Schrödinger and Dirac Equations with Singular Potentials, Vol. 62 (Springer, New York, 2012).

[56] Y. Luke, The Special Functions and Their Approximations, Mathematics in Science and Engineering (Elsevier Science, New York, 1969).

[57] See Supplemental Material at http://link.aps.org/ supplemental/10.1103/PhysRevD.97.061701 for details relative to the eigenvalue spectrum of the Hamiltonian $\hat{H}_{N}$ in Eq. (1), the cutoff regularisation procedure and an explicit expression of the constant $\alpha_{N}$ in Eq. (9).

[58] We have also assumed that the $\Delta_{i}$ do not differ by integer powers to avoid the complication of logarithmic terms in the Frobenius series represented by (3).

[59] In fact, for $\lambda_{N}$ small enough the $\Delta_{i}$ are real and one can find isolated bound states for some choices of the parameter $U(N)$.

[60] The Hamiltonian is to be made self-adjoint in the portion of the half-line considered.

[61] D. Gitman, I. Tyutin, and B. Voronov, Self-adjoint Extensions in Quantum Mechanics: General Theory and Applications to Schrödinger and Dirac Equations with Singular Potentials: 62 (Progress in Mathematical Physics) (Birkhauser, 2012). 